\title{
Prediction of Anomalous Blood Viscosity in Confined Shear Flow
}

\author{
Marine Thiébaud, ${ }^{1, *}$ Zaiyi Shen, ${ }^{1}$ Jens Harting, ${ }^{2,3}$ and Chaouqi Misbah ${ }^{1, *}$ \\ ${ }^{1}$ Université Grenoble I/CNRS, Laboratoire Interdisciplinaire de Physique/UMR5588, Grenoble F-38041, France \\ ${ }^{2}$ Department of Applied Physics, Eindhoven University of Technology, P.O. Box 513, 5600 MB Eindhoven, Netherlands \\ ${ }^{3}$ Faculty of Science and Technology, MESA+Institute, University of Twente, 7500 AE Enschede, Netherlands
}

(Received 27 February 2014; revised manuscript received 24 April 2014; published 10 June 2014)

\begin{abstract}
Red blood cells play a major role in body metabolism by supplying oxygen from the microvasculature to different organs and tissues. Understanding blood flow properties in microcirculation is an essential step towards elucidating fundamental and practical issues. Numerical simulations of a blood model under a confined linear shear flow reveal that confinement markedly modifies the properties of blood flow. A nontrivial spatiotemporal organization of blood elements is shown to trigger hitherto unrevealed flow properties regarding the viscosity $\eta$, namely ample oscillations of its normalized value $[\eta]=\left(\eta-\eta_{0}\right) /\left(\eta_{0} \phi\right)$ as a function of hematocrit $\phi\left(\eta_{0}=\right.$ solvent viscosity). A scaling law for the viscosity as a function of hematocrit and confinement is proposed. This finding can contribute to the conception of new strategies to efficiently detect blood disorders, via in vitro diagnosis based on confined blood rheology. It also constitutes a contribution for a fundamental understanding of rheology of confined complex fluids.
\end{abstract}

DOI: 10.1103/PhysRevLett.112.238304

PACS numbers: 83.80.Lz, 83.50.Ha, 87.16.D-, 87.17.Jj

Introduction.-Blood flow in microcirculation is essential for delivery of nutrients and removal of metabolic waste products to or from tissues. These functions are ensured by proper regulation of blood flow down to the capillary level. One of the main factors controlling capillary circulation is microvascular resistance to blood flow. This effect, in spite of extensive investigation, is still to be fully elucidated, and some fundamental issues remain open. Blood is to good approximation a suspension of red blood cells (RBCs). Blood rheology is dictated by dynamics of RBCs and their interaction with blood vessel walls. A significant research effort has been devoted so far to macroscopic rheology [1,2]. Most of the research on rheology in confined geometries has focused on the famous Fahraeus-Lindqvist (FL) effect [3-5] (see recent review [6]), where confinement has been shown to strongly affect the rheology, with a decrease in apparent viscosity as the diameter of a vessel decreases. These advances have not exhausted yet the intricate behavior inherent to rheology of confined blood, as reported in this Letter.

A property that is commonly of interest for nonconfined suspensions is the viscosity as a function of the volume fraction $\phi, \eta(\phi)$. In the dilute regime, i.e., when hydrodynamic interactions between suspended entities can be neglected, $\eta$ takes the generic form $\eta=\eta_{0}\left(1+a_{1} \phi\right)$, where $\eta_{0}$ is the viscosity of the suspending fluid and $a_{1}$ is a quantity (the so-called intrinsic viscosity), that depends, in general, on the properties of the suspension. For example, for rigid particles, $a_{1}$ is just a universal number and is equal to $5 / 2$; this is the famous Einstein result [7,8]. $a_{1}$ was calculated by Taylor [9] for emulsions, and extended to vesicle suspensions (a blood model) quite recently [10]. When the volume fraction increases, hydrodynamic interactions among suspended entities have to be taken into account, leading to an increase of the suspension viscosity [11]. The classical picture is that when the volume fraction approaches the maximal packing, there is "jamming" accompanied by a divergence of the viscosity at the maximum volume fraction $\phi_{m}$ corresponding to close packing. A commonly used phenomenological law for rigid particles is that of Krieger and Dougherty [12] $\eta=\eta_{0}\left(1-\phi / \phi_{m}\right)^{-a_{1} \phi_{m}}$. In nonconfined suspensions, $\eta$ (as well as $[\eta]=\left(\eta-\eta_{0}\right) /\left(\eta_{0} \phi\right)$, the normalized viscosity, to be used hereafter) is a monotonic increasing function of $\phi$. It will be shown here that confinement can lead to a very different behavior.

We consider either 2D vesicles or 3D capsules (both are model systems for RBCs) endowed with shear elasticity mimicking the RBC cytoskeleton (a network of proteins lying beneath the RBC membrane). Research on vesicles, capsules, and RBCs under flow is very active, regarding both their dynamics [10,13-43] and their rheology $[10,20,22,33,36,44-49]$.

Our study reveals an unusual rheological behavior due to confinement: $[\eta]$ exhibits singularities and nonmonotonic evolution with $\phi$. This results from a subtle interplay between interactions among cells and with the walls. We shall make a clear link between the microstructure and the rheological behavior. We shall also point out the relevance of this spatial order to the FL effect.

The model and the method.-A suspension of 2D vesicles or $3 \mathrm{D}$ capsules is sheared between two rigid plane walls located at $y=-W / 2$ and $y=W / 2$. For vesicles, we adopt the boundary integral formulation with a special Green function which vanishes at the walls [46]. In 3D, we apply a lattice Boltzmann or immersed boundary method for capsules [50,51]. Since the 2D and 3D simulations have 
revealed the same overall qualitative features, most results focus on 2D, albeit 3D illustrations are given to complete the presentation. The cell membrane energy density (per unit area) is composed of the bending energy $(\kappa / 2) H^{2}$ (with $H$ the mean curvature and $\kappa$ the bending rigidity modulus) and, in 3D, of the shear elasticity energy $\kappa_{s}\left(I_{1}^{2}+2 I_{1}-I_{2}\right) / 12+\kappa_{\alpha} I_{2}^{2} / 12$, where $\kappa_{s}$ is the shear elastic modulus and $\kappa_{\alpha}$ is the area dilation modulus. $I_{1}$ and $I_{2}$ are the in-plane strain invariants (see Ref. [50]). $\kappa_{\alpha} / \kappa_{s}=200$ is chosen large enough to preserve local area conservation. We define (in 2D) the reduced area $\tau \equiv$ $(A / \pi) /(p / 2 \pi)^{2}$ (with $p$ the perimeter and $A$ the enclosed area) - taken here to be 0.7 - and the reduced volume (in 3D) $\tau^{\prime} \equiv[V /(4 \pi / 3)] /[A / 4 \pi]^{3 / 2}(V$ and $A$ are the actual volume and the area of the cell), taken to be 0.9 . The ambient and the encapsulated fluids have the same viscosity $\eta_{0}$. A key ingredient in the reported results is the tanktreading (TT) motion of cells. Viscosity of the hemoglobin solution is about 5 times that of the plasma, leading to tumbling (TB) of RBCs. In vitro, TT motion of RBCs is observed when the suspending fluid has a high enough viscosity $\eta_{0}$ [43]. Furthermore, confinement favors TT [52] even with lower $\eta_{0}$. To achieve a basic understanding of rheology, we consider a linear shear flow $v_{x}^{0}=\dot{\gamma} y$ where $\dot{\gamma}$ is the imposed shear rate. Periodic boundaries are used along $x$ and $z$ (in 3D). We define the usual dimensionless numbers (capillary numbers) as $C_{a}=\eta_{0} \dot{\gamma} R_{0}^{3} / \kappa \equiv \dot{\gamma} \tau_{c}$ and $C_{s}=\eta_{0} \dot{\gamma} R_{0} / \kappa_{s}$ with $R_{0}=\sqrt{A / \pi}$ (in 2D) or $R_{0}=$ $[V /(4 \pi) / 3]^{1 / 3}$ (in 3D). In most simulations, $C_{a}=1$ and $C_{s}=0.14$ and the suspended entities exhibit TT motion.

We first focus on the 2D case. The box size in the $x$ direction, $L_{x}$, has to be adequately selected (as discussed in [46]). The relative viscosity and the normal stress difference, $N \equiv\left\langle\sigma_{y y}\right\rangle-\left\langle\sigma_{x x}\right\rangle$, can be expressed in terms of an integral over the vesicles present in the suspension $[46,53]$. We use the (adequate) normalized normal stress difference $[N] \equiv N /\left(\eta-\eta_{0}\right) \dot{\gamma}$ that compares the normal stress to the shear one induced by the suspended entities. The area fraction is varied in the interval $\phi=0 \%-40 \%$.

The rheological properties as functions of concentration.-The first quite peculiar discovery of this study is the nonmonotonic behavior of $[\eta]$ with $\phi$ (Fig. 1). At small $\phi\left(\phi \leq 6 \% \equiv \phi_{\text {tr }}\right),[\eta]$ reduces to the intrinsic viscosity, which is a constant (plateau in Fig. 1). At larger $\phi$ $(\phi>7 \%),[\eta]$ decreases in a quasilinear manner. In the interval $\phi \in[0,15 \%]$ (dilute and semidilute regimes), $[\eta] \simeq$ $a_{1}+a_{2}\left(\phi-\phi_{\text {tr }}\right) Y\left(\phi-\phi_{\text {tr }}\right)$ [54] with $a_{1} \simeq 2.2, a_{2} \simeq-6$, and $Y$ the Heaviside function. This is to be contrasted with $a_{2}=+5$ for nonconfined suspensions [11]. This means that confinement has dramatically altered the rheological behavior. In addition, $[\eta]$ exhibits an apparent singularity around $\phi=15 \%$.

With initial positions chosen randomly within the channel, vesicles first experience a lift force [55-57] due

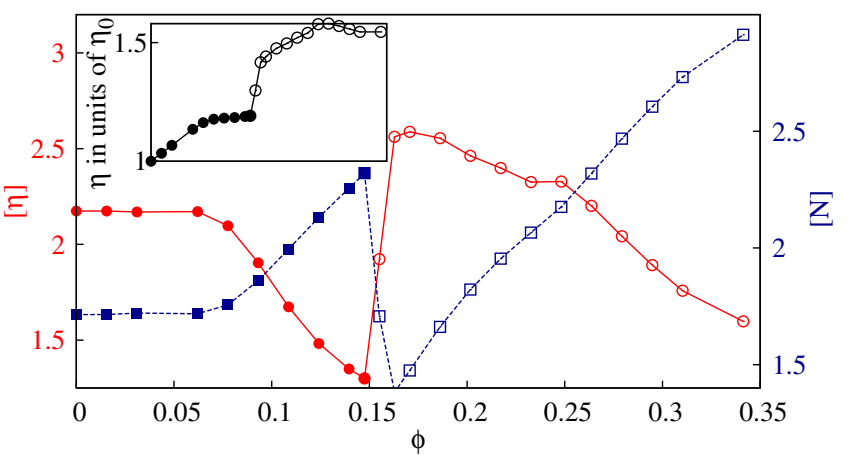

FIG. 1 (color online). The normalized viscosity $[\eta]$ and the normalized normal stress difference $[N]$ as functions of concentration $\phi$ for a channel gap $W=5 R_{0}, R_{0}=\sqrt{A / \pi}$, where $A$ is the vesicle area enclosed by its contour. The left and right $y$ axes denote $[\eta]$ and $[N]$, respectively. The inset reports the viscosity $\eta$. Filled symbols correspond to a single file configuration, while empty symbols correspond to a two-file configuration.

to walls. Because of symmetry, the vesicles stop at the center, exhibiting an ordered alignment along a single file (Fig. 2). The configurations tend to a stationary state, and so does the viscosity of the suspension. At a large enough $\phi$ $(\phi>7 \%)$, vesicles interact with their neighbors. This regime can be referred to as the semidilute regime. Recirculation takes place expressed by vortices between vesicles (Fig. 2). Insertion of additional vesicles (i.e., an increase of $\phi$ ) destroys the large amplitude vortex in favor of a vortex with a weaker amplitude. In other words, an increase of $\phi$ lowers the degree of recirculation. Figure 2(c) shows the quasisuppression of the vortex amplitude [to be contrasted with Fig. 2(a)]. This explains the collapse of dissipation (or $[\eta]$ ) with $\phi$.

Beyond a certain value of $\phi$, the central file [Fig. 2(c)] becomes saturated and the insertion of additional vesicles is hindered due to increased dissipation in the gap separating two successive vesicles, where each membrane tank-treads in opposite directions. Dynamics reveals a spontaneous

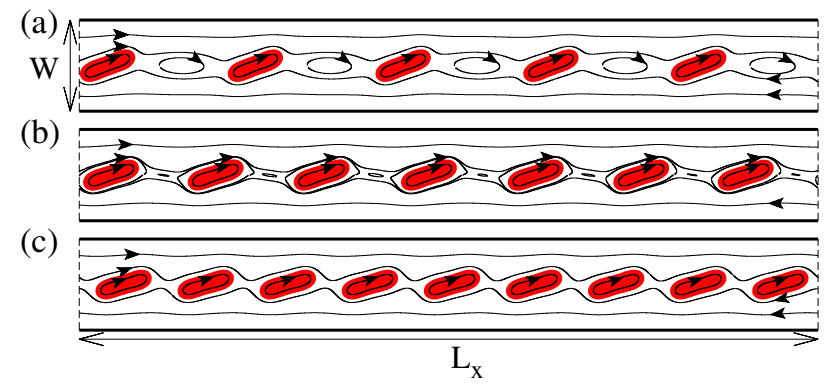

FIG. 2 (color online). The final suspension configurations for the same channel gap as in Fig. 1. Streamlines are shown in black continuous lines. The black arrows indicate the direction of the flow velocity. (a) $\phi=8 \%$, (b) $\phi=11 \%$, and (c) $\phi=14 \%$. Each configuration is a steady one where each vesicle has a fixed position and orientation angle, and each membrane undergoes clockwise tank-treading motion. 


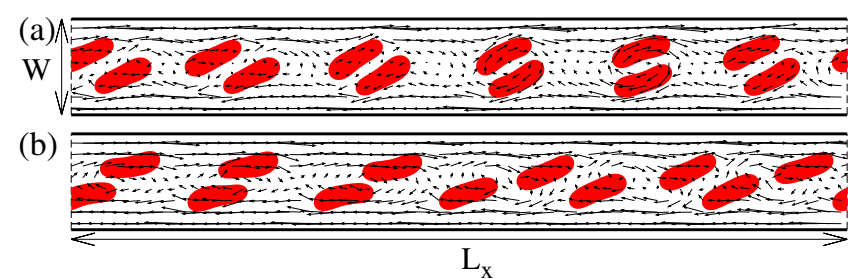

FIG. 3 (color online). A typical vesicle configuration; $W=5 R_{0}$ and $\phi=19 \%$. (a) The vesicles in the top (bottom) file are collectively moving to the right (left) and each vesicle center of mass undergoes oscillations upward and downward in the course of time, while the membrane tank-treads. (b) The configuration at a later time showing the sliding of each vesicle and the vertical motion of their center of mass. The velocity field is shown, but not streamlines which are only suitable for steady configurations; otherwise this could convey the wrong impression that fluid crosses the membranes.

bifurcation of the spatial organization. The suspension undergoes a self-regulating mechanism whereby the initial file splits into two files disposed in a symmetric manner with respect to the flow center line (Fig. 3). The two files undergo a relative countersliding motion. Before this transition, occurring at $\phi \simeq 15 \%,[\eta] \simeq 1.3$, while just after, at $\phi=16 \%,[\eta] \simeq 2.5$, corresponding to a sudden increase of about $90 \%$. In this sense, $[\eta]$ exhibits a pseudosingularity. The bifurcation from a single file towards two files is abrupt and corresponds to a subcritical bifurcation. The details of the precise nature of bifurcations are not a focus of this Letter.

Additional vesicles will be inserted within one or the other file, by keeping symmetry, with some occasional structural defects (not shown here). The insertion of any additional vesicle is accompanied with a decrease of recirculations (decrease of vortices amplitude), and this leads to a collapse of $[\eta]$, as shown in Fig. 1. This collapse occurs in the regime $\phi \in[16 \%, 35 \%]$. Larger $\phi$ leads to the transition from two to three files, with a sudden increase of $[\eta]$, and so on. The three-file (see the Supplemental Material [58]) organization for $W=7 R_{0}$ occurs at $\phi=22 \%$ and survives for more than $100 \tau_{c} \cdot[N]$ (Fig. 1) is positive, meaning that the suspension expands (or swells). $[N]$ also shows a singularity at the two-file transition point, $\phi \simeq 16 \%$.

The rheological properties as functions of confinement.-We have investigated other confinements: $W=3 R_{0}, 4 R_{0}$, and $7 R_{0}$. For $W=4 R_{0}$ or $7 R_{0}$, we observe the same trend as in Fig. 1 . For $W=4 R_{0}$, the abrupt jump of $[\eta]$ is much more amplified as compared to $W=5 R_{0}-$ variation of $[\eta]$ is $>150 \%$-while it is lower $(\simeq 50 \%)$ at $W=7 R_{0}$.

Actually, the overall picture is more complex than presented above, as the results for $W=3 R_{0}$ show. At small $\phi,[\eta]$ shows a plateau, then a decrease and an increase with $\phi$ (Fig. 4), as reported above. However, no new decrease at higher $\phi$ is found. Although two vesicle files could fit into the channel giving rise to the above scenarios,

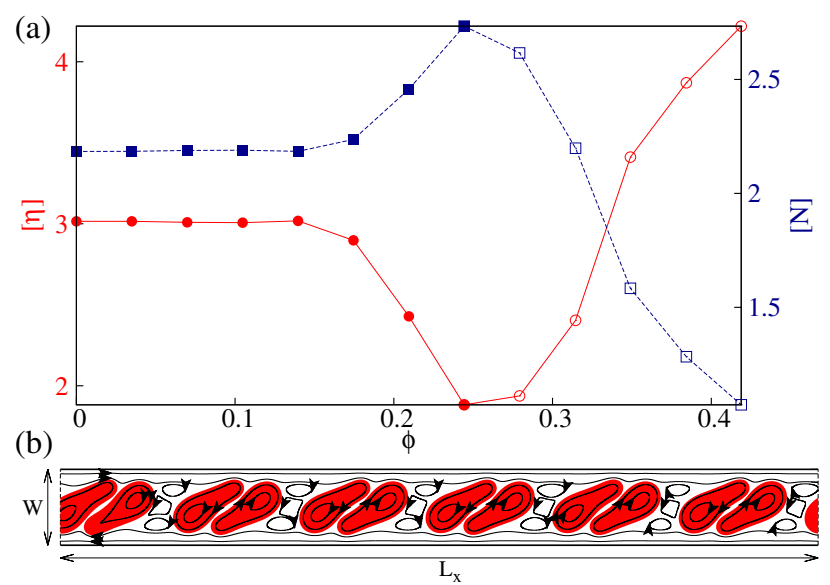

FIG. 4 (color online). (a) The normalized viscosity (left $y$ axis) and the normal stress difference (right $y$ axis) as functions of $\phi$. (b) The final configuration for $W=3 R_{0}$ at $\phi=42 \%$. Streamlines are also shown.

the ordered single file becomes unstable at a critical $\phi$, not in favor of the formation of two parallel files (as observed for other confinements), but by forming doublets of vesicles instead. This attractor of dynamics is accompanied by an enhanced recirculation (Fig. 4), leading to an increase of $[\eta]$. The doublet transition is continuous (supercritical bifurcation). The doublet morphology bears a resemblance to RBC rouleaux caused by adhesion forces $[59,60]$, albeit
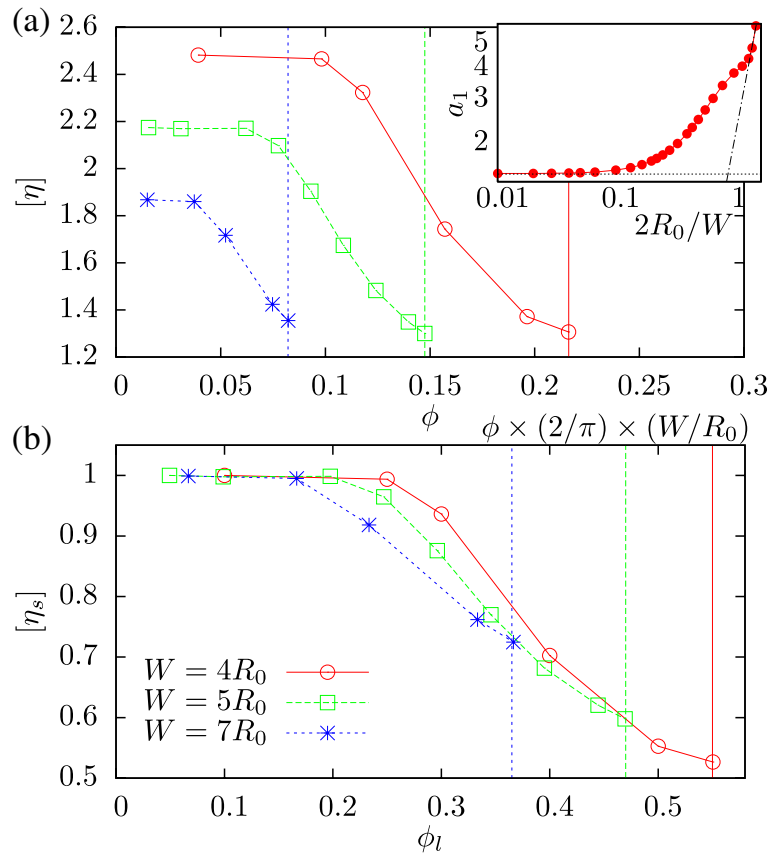

FIG. 5 (color online). Top: $[\eta]$ as a function of $\phi$ for different channel gaps $W$. Inset: $a_{1}$ (intrinsic viscosity) as a function of the confinement $2 R_{0} / W$. Bottom: rescaled data according to Eq. (1) shown for the dilute regime up to the concentration where $[\eta]$ shows a singularity as that shown in Fig. 5(a). 


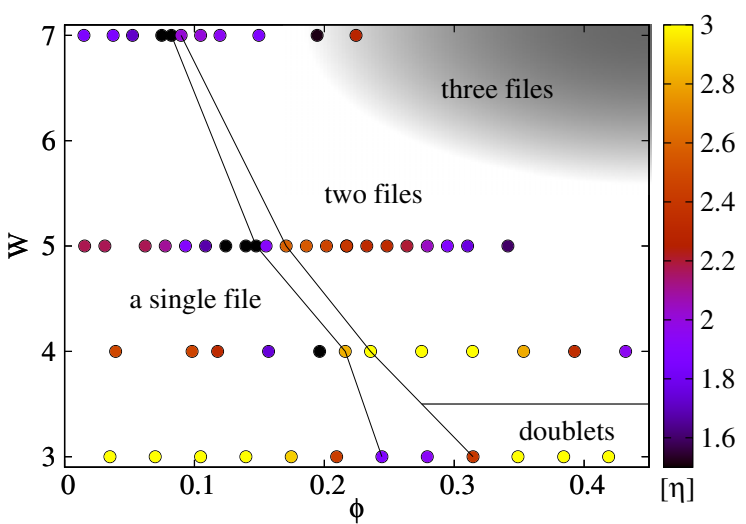

FIG. 6 (color online). The organization diagram. Preliminary simulations also revealed three-file organization.

here it results from membrane deformation under confined flow. In addition, each vesicle "sees" its image through bounding walls. This leads to the fact that $[\eta]$ is not linear even for small $\phi$. Note that in Fig. 3 even if the two files slide with respect to each other, one can identify at every instant also a doublet structure.

Scaling properties.-An appropriate quantity is not $\phi$ itself, but rather the fraction along the flow direction, denoted as $\phi_{l}$. It corresponds to the ratio of the typical diameter of a cell over the available volume per cell along the flow direction, $\phi_{l}=2 R_{0} /\left(L_{x} / N_{\text {ves }}\right)=2 N_{\text {ves }} R_{0} / L_{x}$, and is related to $\phi$ by $\phi_{l}=2 N_{\text {ves }} R_{0} / L_{x}=(2 / \pi) \times$ $\left(W / R_{0}\right) \times \phi$. The transition between the semidilute and the concentrated regimes occurs when cells have no longer enough room along the flow direction which precludes their insertion within the preexisting file. The saturation of the train of cells occurs at approximately $L_{x} /\left(N_{\text {ves }}\right) \sim$ $2 \times\left(2 R_{0}\right)$ (corresponding to $\phi_{l} \sim 0.5$, in accord with the results in Fig. 5). Furthermore, at small enough $\phi,[\eta]$ has a $W$-dependent plateau value $a_{1}(W)$. This suggests the following scaling:

$$
\left[\eta_{s}\right]\left(\phi_{l}\right)=\frac{[\eta]\left(\frac{\phi W}{R_{0}}\right)}{a_{1}\left(\frac{W}{R_{0}}\right)},
$$

where $[\eta]$ and $a_{1}$ are functions of $\phi W / R_{0}$ and $W / R_{0}$, respectively. How $a_{1}$ behaves with confinement is shown in the inset of Fig. 5. The rescaled results reveal a quite reasonable data collapse in the dilute and semidilute regime, as depicted in Fig. 5. The situation turns out to be more complex at higher concentrations, and no simple scaling with confinement could be inferred yet.

Organization diagram.-The bifurcation from the single to the two-file regime (Fig. 6) requires a lower critical $\phi$ for wide gaps $W$ than for narrow ones. This is due to the geometry but also due to the range of hydrodynamic interactions (which increases with $\sim W$ ), favoring collective effects. This implies that a suspension with a certain gap

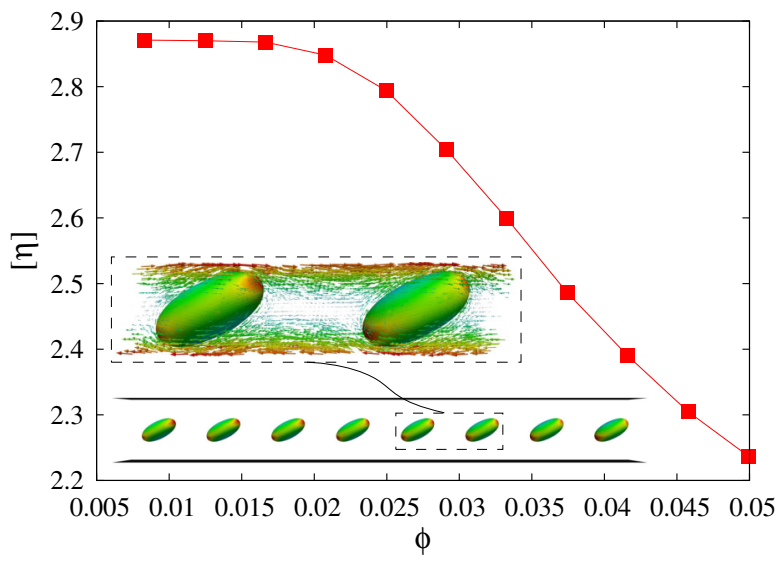

FIG. 7 (color online). $[\eta]$ as a function of $\phi$ and the spatial configuration for $\phi=3.3 \%$. Each capsule is steady (center of mass fixed in time) and the membrane undergoes tank-treading motion, with the velocity field visible on the magnified capsules.

$W_{1}$ may be in the regime of a single file (where $[\eta]$ decreases with $\phi$; see Fig. 5), while a suspension with larger gap $W_{2}$ is entering a two-file regime (sudden increase of $[\eta]$, at the end point of Fig. 5, top). This implies (for the same $\phi$ ) that the viscosity for a smaller gap can be lower than that with a larger gap. This is reminiscent of the FL effect [3]. However, the FL effect is attributed to a depletion layer (cell-free layer) close to the tube wall in a pipe flow. The present effect is rather a consequence of spatial order. This may suggest that the FL effect is not only a result of depletion, but also of spatial organization.

The oscillation amplitude of $[\eta](\phi)$ decreases as $W$ increases, vanishing at a critical $W=W_{c}$, corresponding to a transition from microrheology (oscillatory behavior of $[\eta]$ ) to the traditional macroscopic rheology. A preliminary study suggests $W_{c} \sim 20$.

To confirm the picture, we conduct a systematic analysis in $3 \mathrm{D}$, on which we provide here only a brief account. The system size is given by $L_{x}=40.5 R_{0}, W=5 R_{0}$, and $L_{z}=5 R_{0}$. The 3D rheology follows the same trends as in $2 \mathrm{D}$, conferring to the present results a robust character. Figure 7 shows $[\eta]$ as a function of $\phi$ and the corresponding spatial organization. Note that both the absolute value and the amplitude of viscosity decrease are comparable to those obtained in 2D.

Concluding remarks.-Tumbling is expected to lead to a monotonic increase of $[\eta]$. Several pathologies, such as malaria and sickle cell diseases [61], result in an enhanced stiffness of RBCs. As a consequence, for shear rates where normal RBCs exhibit TT, infected cells can undergo TB. New device conceptions for the detection of blood disorders may use this information. The generality of this behavior for other suspensions is still unclear. A similar impact of confinement on $[\eta]$ for small $\phi$ was reported for a confined rigid sphere suspension [62-64], but neither 
ordering nor oscillation of $[\eta]$ with $\phi$ has been revealed so far.

We thank B. Kaoui and T. Krüger for fruitful discussions. Financial support from CNES, ESA, and NWO/STW is acknowledged. CPU time was provided by the Jülich Supercomputing Centre.

*marine.thiebaud@ujf-grenoble.fr †chaouqi.misbah@ujf-grenoble.fr

[1] S. Chien, Annu. Rev. Physiol. 49, 177 (1987).

[2] Y. Fung, Biomechanics (Springer, New York, 1990).

[3] A. Pries, D. Neuhaus, and P. Gaehtgens, Am. J. Physiol. Heart Circ. Physiol. 263, H1770 (1992).

[4] A. S. Popel and P. C. Johnson, Annu. Rev. Fluid Mech. 37, 43 (2005).

[5] A. R. Pries, T. W. Secomb, and P. Gaehtgens, Cardiovasc. Res. 32, 654 (1996).

[6] T. W. Secomb and A. R. Pries, C.R. Phys. 14, 470 (2013).

[7] A. Einstein, Ann. Phys. (Berlin) 324, 289 (1906).

[8] A. Einstein, Ann. Phys. (Berlin) 339, 591 (1911).

[9] G. Taylor, Proc. R. Soc. A 138, 41 (1932).

[10] C. Misbah, Phys. Rev. Lett. 96, 028104 (2006).

[11] G. K. Batchelor and J. T. Green, J. Fluid Mech. 56, 375 (1972).

[12] I. M. Krieger and T. J. Dougherty, Trans. Soc. Rheol. 3, 137 (1959).

[13] V. Kantsler and V. Steinberg, Phys. Rev. Lett. 95, 258101 (2005).

[14] M.-A. Mader, V. Vitkova, M. Abkarian, A. Viallat, and T. Podgorski, Eur. Phys. J. E 19, 389 (2006).

[15] J. Deschamps, V. Kantsler, E. Segre, and V. Steinberg, Proc. Natl. Acad. Sci. U.S.A. 106, 11444 (2009).

[16] J. Deschamps, V. Kantsler, and V. Steinberg, Phys. Rev. Lett. 102, 118105 (2009).

[17] S. Guido and G. Tomaiuolo, C.R. Phys. 10, 751 (2009).

[18] G. Tomaiuolo and S. Guido, Microvasc. Res. 82, 35 (2011).

[19] G. Coupier, A. Farutin, C. Minetti, T. Podgorski, and C. Misbah, Phys. Rev. Lett. 108, 178106 (2012).

[20] P. M. Vlahovska and R. S. Gracia, Phys. Rev. E 75, 016313 (2007).

[21] V. V. Lebedev, K. S. Turitsyn, and S. S. Vergeles, Phys. Rev. Lett. 99, 218101 (2007).

[22] G. Danker, T. Biben, T. Podgorski, C. Verdier, and C. Misbah, Phys. Rev. E 76, 041905 (2007).

[23] J. M. Skotheim and T. W. Secomb, Phys. Rev. Lett. 98, 078301 (2007).

[24] A. Farutin, T. Biben, and C. Misbah, Phys. Rev. E 81, 061904 (2010).

[25] J. Beaucourt, T. Biben, and C. Misbah, Europhys. Lett. 67, 676 (2004).

[26] H. Noguchi and G. Gompper, Proc. Natl. Acad. Sci. U.S.A. 102, 14159 (2005).

[27] H. Noguchi and G. Gompper, Phys. Rev. Lett. 98, 128103 (2007).

[28] E. Lac, A. Morel, and D. Barthès-Biesel, J. Fluid Mech. 573, 149 (2007).

[29] D.-V. Le and Z. Tan, J. Comput. Phys. 229, 4097 (2010).

[30] S. K. Doddi and P. Bagchi, Phys. Rev. E 79, 046318 (2009).
[31] S. K. Veerapaneni, D. Gueyffier, D. Zorin, and G. Biros, J. Comput. Phys. 228, 2334 (2009).

[32] T. Biben, A. Farutin, and C. Misbah, Phys. Rev. E 83, 031921 (2011).

[33] J. Clausen and C. K. Aidun, Phys. Fluids 22, 123302 (2010).

[34] G. Boedec, M. Leonetti, and M. Jaeger, J. Comput. Phys. 230, 1020 (2011).

[35] H. Zhao and E. Shaqfeh, J. Fluid Mech. 674, 578 (2011).

[36] D. A. Fedosov, W. Pan, B. Caswell, G. Gompper, and G. E. Karniadakis, Proc. Natl. Acad. Sci. U.S.A. 108, 11772 (2011).

[37] A. Z. K. Yazdani and P. Bagchi, Phys. Rev. E 84, 026314 (2011).

[38] P. M. Vlahovska, Y. N. Young, G. Danker, and C. Misbah, J. Fluid Mech. 678, 221 (2011).

[39] Y. Kim and M.-C. Lai, Phys. Rev. E 86, 066321 (2012).

[40] Y. Kim and M.-C. Lai, J. Comput. Phys. 229, 4840 (2010).

[41] W. R. Dodson and P. Dimitrakopoulos, Phys. Rev. Lett. 101, 208102 (2008).

[42] W. R. Dodson and P. Dimitrakopoulos, Phys. Rev. E 85, 021922 (2012).

[43] T. M. Fischer and R. Korzeniewskia, J. Fluid Mech. 736, 351 (2013).

[44] H. Zhao and E. Shaqfeh, J. Fluid Mech. 725, 709 (2013).

[45] A. Lamura and G. Gompper, Europhys. Lett. 102, 28004 (2013)

[46] M. Thiébaud and C. Misbah, Phys. Rev. E 88, 062707 (2013).

[47] P. Bagchi and R. M. Kalluri, Phys. Rev. E 81, 056320 (2010).

[48] V. Vitkova, M.-A. Mader, B. Polack, C. Misbah, and T. Podgorski, Biophys. J. 95, L33 (2008).

[49] V. Kantsler, E. Segre, and V. Steinberg, Europhys. Lett. 82, 58005 (2008).

[50] T. Krüger, F. Varnik, and D. Raabe, Comput. Math. Appl. 61, 3485 (2011).

[51] T. Krüger, S. Frijters, F. Günther, B. Kaoui, and J. Harting, Eur. Phys. J. Spec. Top. 222, 177 (2013).

[52] B. Kaoui, T. Krüger, and J. Harting, Soft Matter 8, 9246 (2012).

[53] G. Batchelor, J. Fluid Mech. 41, 545 (1970).

[54] This raises the question of whether or not a two-body interaction regime exists.

[55] I. Cantat and C. Misbah, Phys. Rev. Lett. 83, 880 (1999).

[56] S. Sukumaran and U. Seifert, Phys. Rev. E 64, 011916 (2001).

[57] T. W. Secomb, B. Styp-Rekowska, and A. R. Pries, Ann. Biomed. Eng. 35, 755 (2007).

[58] See the Supplemental Material at http://link.aps.org/ supplemental/10.1103/PhysRevLett.112.238304 for configurations for different times of the three-file organization.

[59] P. Steffen, C. Verdier, and C. Wagner, Phys. Rev. Lett. 110, 018102 (2013).

[60] C. Wagner, P.Steffen, and S.Svetina, C.R.Phys. 14,459(2013).

[61] S. Suresh, J. Mater. Res. 21, 1871 (2006).

[62] Y. Davit and P. Peyla, Europhys. Lett. 83, 64001 (2008).

[63] P. Peyla and C. Verdier, Europhys. Lett. 94, 44001 (2011).

[64] A. S. Sangani, A. Acrivos, and P. Peyla, Phys. Fluids 23, 083302 (2011). 